\title{
Migración rural a Canadá en México
}

IBIS SEPÚLVEDA GONZÁLEZ

> Universidad Autónoma Chapingo, Texcoco, México. ibisss9@gmail.com

Universidad de Valparaíso

Facultad de Arquitectura

Revista Márgenes

Espacio Arte Sociedad

Migración rural a Canadá en México

Septiembre 2016 Vol. 13 Nº 18

Páginas 26 a 32

ISSN elec. 0719-4463

ISSN imp. 0718-4034

Recepción: enero 2016

Aceptación: abril 2016

\section{RESUMEN}

Migrar al Norte se presenta como la opción ante la pobreza rural, el desempleo y la violencia. Mediante el Programa de Trabajadores Agrícolas Temporales (PTAT) México brinda fuerza de trabajo a Canadá en las cantidades y con los estándares de calificación que necesita para mantener su producción e incrementar su competitividad en el mercado mundial. Recibe poco a cambio: en 2010 mientras Canadá obtuvo de su agricultura más de 21.000 millones de dólares canadienses (CAD) atribuidos en gran medida al PTAT, México recibió remesas por 50 millones (0,24\%). Actualmente Canadá contrata trabajadores temporales de cualquier parte del mundo. Para competir, el gobierno mexicano capacita más a los migrantes y negoció que paguen por agua, gas y electricidad. Los jornaleros no saben si al año siguiente serán llamados. En caso favorable, se enfrentan a condiciones de vida y trabajo que distan de ser adecuadas. Regresan con ahorros que serían imposibles en México, pero sus opciones de invertirlos productivamente son muy limitadas, pues comen de allí en la temporada intermigratoria. Desean invertir en microempresas, pero requieren recursos adicionales. Estamos trabajando para apoyarlos en organización y creación de pequeños negocios a contracorriente.

PALABRAS CLAVE

jornaleros agrícolas, migración laboral internacional, microempresas rurales

\section{Rural migration to Canada in Mexico}

ABSTRACT

Migrating north is presented as the solution to rural poverty, unemployment and violence. Through the Temporary Agricultural Workers Program (PTAT) Mexico provides workforce to Canada in the amounts and standards it needs in order to maintain its production and increase its competitiveness in the global market. Mexico receives little in exchange: while in 2010 Canada received 21,000 million Canadian dollars (CAD) from its agricultural activity, most of it attributed to PTAT, Mexico received an income of 50 million (0,24\%). Canada currently hires temporary workers from all around the world. In order to compete, the mexican government its migrant workers even more and negotiated the oayment of water, fuel and electricity. Workers don't know if they'll be called next year. Best case scenario, they face far from suitable working and living conditions. They return with savings that would be impossible to acquire in Mexico, but their chances to productively invest these savings are very limited, since these savings provide food during the intermigratory season. They wish to invest in small enterprises, but they require additional resources. We're working to help them organize and create small enterprises against the grain.

KEYWORDS

agricultural laborers, international labor migration, small rural enterprises 


\section{INTRODUCCIÓN}

La migración internacional es un fenómeno de múltiples causas y efectos. En este caso de migración laboral controlada y legal, el fenómeno es parte de la globalización que en los países periféricos erosiona los medios tradicionales de subsistencia y deja a las clases trabajadoras sólo el recurso de emigrar, pues escasean los puestos de trabajo en sus países de origen. Estas migraciones laborales permiten mucha más acumulación de riqueza en los países ricos, debido a que el trabajo de los migrantes es significativamente más barato que el de los nacionales. Además de facilitar la acumulación privada, los migrantes internacionales no significaron para el país receptor un gasto en su formación y este tampoco se hace responsable por el mantenimiento ni por la reproducción de la fuerza laboral bajo las condiciones "normales" del país (Canadá en este caso), pues año con año se la surten otros, México en el caso discutido, lista para el trabajo. De esta manera, la globalización se nutre no sólo de flujos de capital, sino también de flujos de fuerza de trabajo.

En Canadá, Thomas (1997) resalta la diferencia entre la categoría de "migrante" (migrant) e "inmigrante" (inmigrant) puesto que el segundo tiene el derecho de residencia y de optar por la ciudadanía, mientras que el migrante sólo tiene un estatus de residente temporal, sin ninguna posibilidad de pretender hacerse ciudadano. Así, no tiene libertad de circular en el mercado laboral, ni ninguna de las garantías que el estado canadiense otorga a sus ciudadanos.

A partir de 2002 Canadá está importando más trabajadores temporales para todas las ramas de su economía, provenientes de distintos lugares del mundo, mediante el Proyecto Piloto (NOC C \& D) para fuerza de trabajo de baja calificación. Este proyecto, en el cual se disminuye el papel de los gobiernos involucrados y los migrantes temporales quedan atenidos a sus propios recursos, parece que será el principal instrumento de migración laboral a Canadá, claro, en una enorme competencia de fuerza de trabajo internacional.

Así, son los países desarrollados los que marcan el sentido, las condiciones y la magnitud de los flujos laborales internacionales. Pero no hay que minimizar la importancia de los factores de expulsión nacionales que complementan estas migraciones.

\section{SOBRE EL PROGRAMA DE TRABAJADORES AGRÍCOLAS TEMPORALES MÉXICO - CANADÁ}

El Programa de Trabajadores Agrícolas Temporales (PTAT) México - Canadá es un instrumento que proporciona mano de obra de un país con una población abundante (120 millones de habitantes) a otro cuya pujante economía pero débil tasa de crecimiento demográfico y relativamente baja población $(0,79 \%$ y 34 millones de habitantes), lo hace necesitar más fuerza de trabajo que la suya propia.

Este Programa ha sido considerado un modelo de migración laboral internacional. En el plano internacional se ha dicho que es un flujo ejemplar de mano de obra. Algunos países han mostrado interés por llevarlo a cabo como parte de su política migratoria. Australia y la Comunidad Europea han estudiado su funcionamiento para regular el trabajo de ciudadanos extranjeros y como una medida efectiva para enfrentar la reducción de la fuerza laboral en sus respectivos mercados de trabajo domésticos.

El Programa tuvo su origen en 1974 como un acuerdo administrativo intergubernamental no considerado como tratado internacional, el Memorándum de Entendimiento (ME) entre el Gobierno de los
Estados Unidos Mexicanos y el Gobierno de Canadá relativo al Programa de Trabajadores Agrícolas Mexicanos Temporales y el Acuerdo para el Empleo Temporal de Trabajadores Agrícolas Mexicanos en Canadá. Este último es un instrumento que los trabajadores, sus empleadores y el Agente Gubernamental respectivo (usualmente los cónsules de México en Canadá) deben firmar individualmente. En el ME, refrendado en abril de 1995, los gobiernos destacan que el PTAT simboliza los vínculos estrechos de amistad, comprensión y cooperación entre ambos países (SRE, 2010).

La administración del Programa por parte de Secretarías de Estado y organismos privados Foreign Agricultural Resource Management Services (FARMS/FERME), es una característica del modelo PTAT. El argumento es que el PTAT responde a una demanda y una oferta de los mercados laborales agrícolas entre ambos países. Lo sintetiza el objetivo mexicano: Generar una movilidad laboral anclada en las necesidades del mercado del país de origen y destino, evitando desequilibrios y generando complementariedad en los mercados laborales de ambos países (STPS, 2012).

Sus objetivos, según se establece en los documentos oficiales del Programa, son:

- Satisfacer la demanda temporal de los productores de hortalizas de trabajadores agrícolas poco calificados durante las temporadas de plantación y cosecha, siempre que exista un déficit de trabajadores canadienses con habilidades similares.

- Ayudar a mantener la prosperidad económica de Canadá y su competitividad agrícola en el comercio mundial a través de la oportuna realización de las tareas de cultivo, cosecha, procesamiento y venta de frutas, verduras y otros productos hortícolas, y ampliar las oportunidades de trabajo para los canadienses que dependen de la agricultura y de las actividades relacionadas con la industria agrícola.

- Incrementar y mantener la eficiencia económica de Canadá mediante la asignación de recursos laborales locales.

- Mejorar el bienestar económico de los trabajadores migrantes y de sus familias, proporcionándoles trabajo temporal de tiempo completo en actividades de uso intensivo de mano de obra de la industria hortícola, con salarios relativamente más altos que los que podrían obtener en sus países por actividades similares o alternas.

- Facilitar el regreso voluntario de los trabajadores a sus países, al terminar su empleo temporal en Canadá (STPS, 2006).

El perfil requerido para los trabajadores del PTAT en 2014, es:

- Ser campesino, jornalero o peón de campo

- Edad entre 22 y 40 años

- Presentar acta de nacimiento del titular y sus beneficiarios

- Identificación vigente del titular y beneficiarios

- Estar casado o vivir en unión libre por más de tres años. Presentar actas.

- Constancia de estudios

- Comprobante de domicilio

- CURP del titular y beneficiarios

- Conocimiento y experiencia en siembra, (y) cosecha de productos de campo, de invernadero y vivero (INMUJERES 2014:42). 
Existen tres categorías de trabajadores: Trabajador nominal, Trabajador de selección y Trabajadores de reserva. Aunque los nominales tienen más posibilidades, ninguno tiene seguridad de volver a ser solicitado el siguiente año.

EI PTAT inicia en 1974 con 203 trabajadores y en una primera etapa crece lentamente, para alcanzar 834 trabajadores en 1985. En un segundo período, de 1986 a 1996, se incrementa el promedio anual de trabajadores enviados hasta 5,000. De 1996 a 2015 el número ha venido en aumento esperando enviar 20.000 en el presente 2015.

La siguiente tabla da cuenta del crecimiento del Programa:

\begin{tabular}{|c|c|}
\hline Año & Número de trabajadores \\
\hline 1975 & 203 \\
\hline 1978 & 495 \\
\hline 1980 & 553 \\
\hline 1982 & 655 \\
\hline 1984 & 615 \\
\hline 1986 & 834 \\
\hline 1987 & 1538 \\
\hline 1988 & 2623 \\
\hline 1989 & 4414 \\
\hline 1991 & 5143 \\
\hline 1993 & 4778 \\
\hline 1995 & 4910 \\
\hline 1997 & 5211 \\
\hline 1998 & 6486 \\
\hline 2000 & 9175 \\
\hline 2003 & 10681 \\
\hline 2005 & 11720 \\
\hline 2006 & 12868 \\
\hline 2007 & 14288 \\
\hline 2008 & 15849 \\
\hline 2010 & 15809 \\
\hline 2011 & 16491 \\
\hline
\end{tabular}

> Fuente: STPS 2012, op. cit. Elaboración propia.

Como se puede observar, es en la década pasada cuando el Programa comienza a crecer de manera más pronunciada en México, a pesar de que ya se había establecido en Canadá el Proyecto PiIoto NOC C y D. Esto permite el supuesto de que aunque el último proyecto presenta las mayores características de liberalización, el PTAT ha demostrado con suficiencia un buen funcionamiento y obediencia a sus lineamientos por parte de los mexicanos, lo que da seguridad a los empleadores.

De acuerdo con el formato de Contrato que tiene el Ministerio de Recursos Humanos y Desarrollo de Competencias de Canadá (HRSDC - Human Resources and Skills Development Canada) en su página web (2012) hay una serie de requisitos que las partes deben cumplir. Entre estos, destacan los siguientes y se señalan hechos que los ponen en duda:

- Que aunque la jornada laboral es de 8 horas, el empleador puede pedir al trabajador que labore más en situaciones de emergencia, hasta un máximo de 12 horas. Sin embargo en una encuesta realizada en 2007 en Tlaxcala dentro del pro- yecto CONACYT aludido, el tiempo promedio que laboraban los trabajadores era de 12 horas diarias (Sepúlveda et al., 2011). Las jornadas se han intensificado, de acuerdo a pláticas recientes con migrantes que señalan hasta 17 horas diarias en temporada pico. En 2012 en una entrevista al periódico La Jornada, la investigadora canadiense de la Universidad de Windsor Dra. Tanya Basok, señaló que los migrantes cumplían jornadas laborales de hasta 16 horas diarias (Poy, L. 2012:17).

- Que el empleador deberá proporcionar gratuitamente al trabajador un alojamiento adecuado y comidas razonables $y$ adecuadas $y$, si el trabajador prepara sus propios alimentos, proporcionarle utensilios de cocina, combustible e instalaciones sin costo alguno para el trabajador (HRSDC, 2012). Sin embargo, a partir de 2012 cada trabajador debe pagar 2,16 dólares canadienses diarios por concepto de combustibles, luz y agua de sus viviendas (STPS, 2012). Esto ya está estipulado en el contrato.

- Que el empleador deberá permitirle al trabajador un mínimo de 30 minutos para la comida y 10 minutos de descanso a media mañana y a media tarde. Este periodo puede o no ser pagado, de acuerdo a la legislación provincial de que se trate. La provincia de Ontario, que recibe al $49 \%$ de los trabajadores, no contempla el pago de este tiempo. Según trabajo de campo se les descuentan del salario estos 50 minutos (o en algunos casos una hora), a la mayoría de los trabajadores mexicanos en Canadá.

- Que se les pagará lo que corresponda al salario corriente para el tipo de trabajo agrícola efectuado por el trabajador en la provincia en que realice el trabajo. Hay que destacar que el salario agrícola es el más bajo que contempla la legislación canadiense.

- $\quad$ Que si por cualquier motivo no fuese posible laborar, el trabajador recibirá un adelanto, con un recibo firmado por el trabajador, para cubrir sus gastos personales; el empleador tendrá derecho a deducir dicho adelanto de la remuneración del trabajador antes de su partida (HRSDC, 2012). Según lo expresado por tabajadores entrevistados en 2012 y 2014 , esto suele suceder a consecuencia de fenómenos metereológicos o por caídas del mercado, de tal manera que aunque no sea responsabilidad del trabajador, es éste el que debe pagar por las interrupciones en el trabajo.

- Que el trabajador debe pagar 0,94 dólares canadienses diarios por concepto de seguro médico no ocupacional. El contrato no es muy claro cuando se refiere a accidentes del trabajo, pero en entrevista realizada a Andrea Galván, representante del sindicato UFCW en México (14 de septiembre de 2011), ella señala que los trabajadores deben ser atendidos por Salud y Seguridad en el Trabajo, programa de gobierno donde el patrón debe pagar por la atención, pero que convencen a los trabajadores de solicitar el seguro privado, actualmente con el Royal Bank of Canada (RBC), donde el empleador paga la prima total y se la descuenta luego a los trabajadores, tal como lo prevé el contrato en la Cláusula V párrafo 3 (HRSDC, Op. Cit. 2012). El RBC cubre el 90\% del salario hasta por seis semanas cuando el trabajador está incapacitado. Pero cuando el trabajador se enferma más seriamente, sencillamente es repatriado. 
- Que por el viaje internacional ida y vuelta, el empleador tiene derecho a deducir hasta 589.00 dólares canadienses, más 150 dólares canadienses por pago de la visa temporal de trabajo, del pago semanal del trabajador. Cuando la autora leyó este ordenamiento, el boleto de Ciudad de México al Aeropuerto Internacional de Ontario por American Airlines para un periodo del 23 de abril al 30 de noviembre de $2012^{1}$, costaba 305 dólares americanos. No resulta fácil explicarse por qué el empleador puede descontar hasta 589 dólares canadienses por boleto, que además es comprado para grupos grandes, cuando en internet para una sola persona en clase turista, puede conseguirse notablemente más barato.

- Que el trabajador debe realizar y pagar su declaración de impuestos, tal como lo establece la ley para cualquier trabajador canadiense. Este es un punto muy polémico, ya que el trabajador temporal paga seguro de desempleo, pero no recibe pensión por los meses de la temporada intermigratoria. También aporta para el Canadian Pension Plan, que paga las jubilaciones de los ciudadanos canadienses, pero en el caso de que logre tramitar su jubilación, hemos encontrado en nuestro trabajo de campo que los jubilados mexicanos reciben alrededor de 100 a 120 dólares canadienses mensuales, después de haber cumplido los 65 años y con más de 100 meses de trabajo en el PTAT.

Según una investigación CONACYT realizada en Tlaxcala en 2006 - 2007 (Sepúlveda et al, 2011), los trabajadores del PTAT de Tlaxcala, que en 2011 fueron $1.925^{2}$, tenían 37 años de edad en promedio; 46\% tenían estudios de secundaria completa o superiores; $82.5 \%$ de ellos tenían experiencia laboral en la agricultura y más del $80 \%$ no tenían otras fuentes de ingreso más que su trabajo en Canadá. Estas condiciones, más un buen estado de salud verificado en México, hacen de estos trabajadores un contingente ideal para un buen cumplimiento laboral.

En la investigación citada cabe remarcar como un hallazgo importante el que los trabajadores PTAT en su gran mayoría, desean invertir sus ahorros en proyectos productivos que les permitan aspirar a un nivel de vida digno una vez que los empleadores canadienses ya no los soliciten. Esto reviste mayor importancia debido a la escasa proporción de trabajadores jubilados del PTAT en los 40 años que tiene de vigencia y a la muy escasa pensión que logran recibir, los pocos que la tramitan (deben hacerlo en Canadá), después de cumplir los 65 años de edad.

De acuerdo a la Secretaría del Trabajo y Previsión Social, se puede caracterizar al PTAT de la siguiente manera:

Es un esquema de movilidad laboral circular, ordenado, legal y seguro.

Permite la articulación de esfuerzos de autoridades estatales y federales de ambos países.

Genera una movilidad laboral anclada en las necesidades del mercado del país de origen y destino, evitando desequilibrios y generando complementariedad en los mercados laborales de ambos países.

Asegura la pertinencia laboral de los trabajadores, tanto para los empleadores como para ambos gobiernos, asegurando tanto la eficiencia como la circularidad y retorno de los trabajadores a México.
Asegura el Trato Nacional para los trabajadores mexicanos, ya que los trabajadores del PTAT tienen los mismos derechos y obligaciones que los trabajadores canadienses.

Garantiza la Portabilidad de beneficios sociales para los trabajadores (antigüedad, derechos, etc.), ya que aportan al sistema de salud y pensión canadiense, lo que asegura la atención médica, pensiones y otros beneficios.

Genera un mercado laboral regional integrado, que impulse la formación de capital humano y genere capacitación y transferencia del aprendizaje laboral (STPS, 2012:2).

Este discurso de la Secretaría del Trabajo de México, que es quien selecciona y facilita el contrato en México, es absolutamente similar al que se maneja en Francia con los contratos OMI (actualmente la Office des Migrations Internationales es I'Office Français de I'Immigration et de I'Intégration - OFII) y en España, como lo apunta Reigada: Por lo general, este sistema de contratación (Sistema de Contratación en Origen) ha tendido a ser definido a partir de las ventajas que crea para el gobierno, preocupado por frenar la inmigración "ilegal"; para el sector empresarial, que ha encontrado en dicho sistema la posibilidad de planificar con suficiente antelación la campaña, y para las trabajadoras, a las que se les ofrecería una oportunidad de mejorar su nivel de vida. No es de extrañar, por tanto, que bajo la idea del "beneficio mutuo", empresarios, administraciones y sindicatos mayoritarios coincidan en considerarlo como el “modelo ideal de la inmigración ordenada (Reigada, 2012:24). Sin embargo, los datos que proporciona Muñoz (2011) al señalar que en 2010 México recibió de Canadá remesas de 50 millones de dólares canadienses, mientras Canadá obtuvo de su agricultura más de 21.000 millones de dólares en el mismo período, atribuidos en gran medida al PTAT, aunque pretendían abonar al citado beneficio mutuo, explicita que en este caso, México sólo recibe las migajas.

\section{FUERZA DE TRABAJO PARA EL FUTURO}

La preocupación por asegurarse fuerza de trabajo para el futuro es un tema importante en los países más desarrollados, como lo evidencia este informe de la Unión Europea: Aunque, hasta cierto punto, la inmigración podrá mitigar los problemas derivados del envejecimiento de la población, tendrá todavía un mayor papel en ayudar a resolver los problemas futuros del déficit de mano de obra y personal cualificado y en aumentar el potencial de crecimiento de la UE y su prosperidad, en complemento de las reformas estructurales en curso. Por ello, la inmigración se ha convertido en un factor importante en el desarrollo de la Estrategia de Lisboa para el crecimiento y el empleo, que reconoce que una gestión adecuada de la inmigración económica es un factor esencial para la competitividad de la UE. Esto también ha sido reconocido por el Consejo Europeo de la primavera de 2008 (Comisión de las Comunidades Europeas, 2008:3).

Por su parte Canadá parecía estar en la misma sintonía al haber establecido para los mexicanos participantes en el PTAT una "ayuda de paternidad" a cambio del pago de seguro de desempleo que a ellos no se les paga. De esta manera, por cada hijo nacido en México a un trabajador que reúna al menos 600 horas de trabajo en el año del nacimiento y que tenga al menos dos temporadas de antigüedad en el Programa, se le otorgaban alrededor de 5.000 dólares canadienses: Me depositaban 250 dólares cada 15 días, por 15 quincenas a partir del nacimiento de mi bebé. Al llegar, me dieron 1.000 dólares más (María G., trabajadora nominal en Ontario. Entrevista 22.03.2012). 
Si bien para algunos migrantes la ayuda de paternidad era un beneficio a cambio de sus impuestos, otros sí lo percibían como una forma de estimular el incremento poblacional en un país que les proporciona mano de obra, pero que no es el suyo: $A$ ellos les conviene que nosotros tengamos hijos. Así, como seguimos siendo pobres, ellos esperan que nuestros hijos sean los que les sirvan cuando nosotros ya no les sirvamos por viejos. Pero a los niños los vamos a tener que seguir manteniendo nosotros (Benito R., trabajador en Ontario. Entrevista 10.04.2012). De esta manera, algunos trabajadores sí han visto en la paternidad una nueva fuente de ingresos, pero otros sacan cuentas más a largo plazo y ven que el "negocio no les conviene". La ayuda parental dejó de tener vigencia en 2013.

\section{Y CUANDO REGRESAN LOS TRABAJADORES DEL PTAT, ¿QUÉ HACEN Y QUÉ DESEAN HACER?}

\section{Lo que hacen}

EI PTAT elige preferentemente a personas que tengan hijos pero que no tengan medios de producción propios. La mayoría son jornaleros agrícolas, albañiles, tienen algún pequeño negocio (miscelánea, cocina económica, taller mecánico) o algo de ganado, generalmente en tierras rentadas o prestadas por familiares. Estos empleos, aunque a menudo son múltiples (pluriactividad), no son permanentes ni seguros ni suelen permitirles un ingreso para vivir por lo que deben ocupar parte considerable de sus ahorros para sobrevivir en el período intermigratorio.

En su gran mayoría, estos migrantes tlaxcaltecas desean invertir sus ahorros en proyectos productivos que les permitan generar un ingreso en el invierno que pasan en México y aspirar a un nivel de vida digno una vez que los empleadores canadienses ya no los soliciten. La edad de los trabajadores solicitados es de hasta 40 años, siendo muy difícil que gente mayor al límite sea contratada, a menos que sean trabajadores nominales de excelencia y sus patrones los soliciten por sus nombres.

En los períodos intermigratorios, que a veces se prolongan por largo tiempo, los migrantes vuelven a buscar las mismas actividades que realizaban antes de migrar. Así, vuelven a andar solicitando alguna clase de trabajo remunerado, o inician cultivos en escasas superficies de tierras prestadas, rentadas o, los menos, en tierras propias. Otros se dedican a cuidar los pocos animales que han logrado adquirir y a construirles corrales rudimentarios.

Muy pocos, que tienen en Canadá trabajos más especializados, como lo es la producción de la miel, trabajan en sus propios emprendimientos, aunque sujetos a robos, como fue un caso notable en este año.

En general, los trabajos remunerados que consiguen, sean propios o de empleadores ajenos, no ingresan los recursos suficientes para mantener a la familia y la mayoría de los migrantes deben echar mano de sus ahorros.

\section{Lo que desean hacer los migrantes}

Los proyectos productivos revisten mayor importancia en el imaginario de los migrantes debido a las pocas expectativas de trabajo e ingresos que tienen a futuro. La escasa proporción de trabajadores jubilados del PTAT en todos los años que tiene de existencia y la muy escasa pensión que logran recibir, es otro argumento para buscar desarrollar alguna actividad propia que ofrezca la esperanza de ser rentable.
De esta manera, nuestro pequeño equipo de investigación y acción ${ }^{3}$ los ha apoyado en el fomento a la idea de crear microempresas y de organizarse para tener una figura propia que los represente ante funcionarios y autoridades mexicanas que tengan capacidad de atender sus demandas ${ }^{4}$. Los proyectos que durante 2014 se propusieron a la Secretaría de Agricultura, Ganadería, Pesca y Alimentación, SAGARPA, a través del Fondo para el Apoyo a Proyectos Productivos en Núcleos Agrarios (FAPPA), se enlistan a continuación. Ellos están dispuestos a arriesgar sus ahorros en los proyectos productivos que proponen, siempre que el Estado los apoye con aproximadamente un 50\% y le proporcione capacitación a su equipo de trabajo. Aunque ellos se van a Canadá, todos tienen familiares adultos dispuestos a hacerse cargo de los proyectos mientras ellos están migrando. La forma del proyecto sería por grupos familiares de seis integrantes, donde desempeñan un papel muy destacado las mujeres, esposas e hijas de los que se van.

Las iniciativas de los migrantes en 2014, por municipio fueron las siguientes:

- $\quad$ En Benito Juárez quieren 11 proyectos: Cinco de producción de borregos para engorda. Dos proyectos de establecimiento de tienda de abarrotes. Establecimiento de un taller de maquila de calzado. Adquisición de un tractor. Producción de cebolla. Establecimiento de un gimnasio.

- En Cuamatzingo, Muñoz de Domingo Arenas, los migrantes están interesados en 7 proyectos: Tres proyectos de producción de borregos para engorda. Dos de establecimiento de un invernadero para la producción de tomate. Adquisición de un tractor. Uno de producción de vacas para leche y carne.

- En San Antonio Atotonilco, Municipio de Ixtacuixtla, hay 16 interesados en proyectos: Nueve proyectos de ganadería. Dos de invernaderos. Dos de comercios. Uno de transporte. Uno de apicultura. Uno de taller de costura.

- En San Lucas Tecopilco hay interés por 5 microempresas: Dos proyectos de ganadería. Uno de una granja de pollos. Uno de apicultura. Uno de ampliar un taller mecánico.

- En el municipio de Panotla hay interés en 1: Un grupo interesado en la cría y engorda de reses.

Como se puede apreciar en los 40 proyectos en cinco municipios donde tenemos presencia, los intereses son variados, pero cabe destacar que la mayoría se inclinan por proyectos de producción agropecuaria y donde sobresalen los pecuarios.

Las facilidades para elaborar los proyectos productivos de acuerdo a las especificaciones señaladas por las instituciones no son las adecuadas pues exigen demasiados detalles, muchos de los cuales no son una garantía de la viabilidad del proyecto sino al parecer, un freno para desarrollar proyectos pequeños de gente con pocos recursos.

Cabe destacar el gran interés que las mujeres, tanto migrantes ellas mismas como esposas e hijas de migrantes, están demostrando en realizar actividades productivas propias.

Sin embargo, durante el año nos enteramos que independientemente de lo bien hecho que pudiera haber estado el proyecto, sólo se aprobaron aquellos presentados por organizaciones sociales y políticas. Por lo tanto, en 2014 no nos aprobaron ninguno de los proyectos presentados. 
Así, en 2015 probamos de pedir el apoyo de la organización Unión Campesina Democrática para que nos permitiera, por su intermedio, solicitar algunos proyectos. Sólo nos dieron opción de un proyecto (Mielissima, de San Lucas Tecopilco), mismo que fue aprobado en julio de 2015.

\section{CONCLUSIONES}

La forma de utilización de la fuerza de trabajo es actualmente un factor clave para el desarrollo de las economías mundiales. Los países con una capacidad económica elevada y con bajas tasas demográficas, buscan satisfacer sus demandas de fuerza de trabajo no con la inmigración definitiva de extranjeros, sino con la utilización temporal de gente joven y capacitada para las labores en que las necesitan. Sus economías son las que marcan el ritmo y la intensidad de las migraciones laborales, integradas por ciudadanos de países pobres cuyas políticas no buscan ocuparlos en sus lugares de origen sino las divisas que pueden obtener de ellos. Cada vez más gente vive de las remesas que los migrantes laborales envían a sus familias, por lo que estas personas están siempre dispuestas a migrar. Los factores de expulsión en los países de origen se han convertido en una constante.

Así, los países ricos que tienen poblaciones escasas, mantienen sus economías crecientes y las prestaciones sociales para su población envejecida, con la fuerza de trabajo barata y temporal de jóvenes de países pobres, que no han desarrollado otra estrategia de desarrollo más que la exportación de brazos, considerados redundantes en sus propios países. Al contrario, emergentes como los BRICS, han probado que el bono demográfico es un factor esencial para el crecimiento económico, al igual que la inversión en ciencia y desarrollo (González, 2008).

Desafortunadamente la clase política de México, no ha mostrado interés consistente en la creación de empleos y sí en las divisas obtenidas por las exportaciones de muchas cosas, entre ellas de fuerza de trabajo, definitiva o al menos, temporal.

De esta manera, sobre todo Estados Unidos, pero también Canadá, son los confines migratorios preferidos de los mexicanos que no consiguen ganarse la vida en su país de origen.

Los dos grandes programas de contratación temporal de fuerza de trabajo extranjera que tiene hoy Canadá, le permite seguir manteniendo sus mercados internos y continuar compitiendo con notable éxito en los mercados globales. Cabe hacer notar que del valor de su producción, en este caso la agrícola que ha estado fundamentalmente en los brazos de los jornaleros mexicanos del PTAT, la porción destinada al pago de la fuerza de trabajo, es ínfima. Así, el argumento de que ambos países ganan con este esquema, resulta engañoso.

El Estado mexicano no se ha preocupado por crear empleos, pero tampoco por mejorar las condiciones laborales de sus migrantes. Al contrario, quiere competir con otros países poco desarrollados que también ven un alivio en la exportación de personas, bajando los ingresos de los mexicanos para que los empresarios los prefieran.

Tanto el PTAT como el Mecanismo de Movilidad Laboral (MML), designación mexicana para el Proyecto Piloto NOC C y D, representan relaciones binacionales inequitativas, en un transnacionalismo normado y prohijado por los respectivos Estados. El Estado mexicano a través del MML no ha querido hacerle la competencia al PTAT al no promocionar en el país la rama agrícola del Proyecto Piloto NOC C y D. Sin embargo, al analizar los salarios que se ofrecen para otro tipo de trabajos a través del MML, se puede observar la misma tendencia: salarios sumamente bajos para los mexicanos. Comparados ambos programas en salarios medios y condiciones $y$ exigencias de los contratos, a pesar de muchos factores objetivamente negativos del PTAT, éste parece resultar una mejor opción de empleo para los mexicanos.

Lo que resulta incuestionable es que los trabajadores migratorios temporales de México, así como sus familias, requieren y merecen fuentes de trabajo dignas en sus lugares de origen. Ellos así lo perciben y están dispuestos a arriesgar sus ahorros logrados con tanto sacrificio, en microempresas familiares en las que también puedan desarrollar habilidades y visión adquiridas en los países del Norte, en este caso, en Canadá.

Esto representa una opción de política de desarrollo regional alternativa que, si fuera asumida y apoyada por el gobierno federal y estatal, podría ser sumamente favorable tanto para la economía de las familias emprendedoras como para la de sus regiones, de su estado y del país.

El reto es muy grande, porque se trata de remar a contracorriente del modelo económico general dominante que favorece a las grandes compañías y que se observa dispuesto a transnacionalizar hasta las riquezas no renovables más paradigmáticas del país. Además, hay que enfrentar la carga burocrática de las instituciones destinadas a aportar apoyos y capacitación a los pequeños emprendedores, pero que en hechos y palabras, manifiestan que los recursos públicos escasos, deben destinarse a los productores que ya tienen recursos propios, para hacer crecer más sus negocios que ya saben manejar.

De esta manera, los riesgos son muchos y el esfuerzo muy grande, pero creemos que la alternativa a esto, el no hacer nada, es condenar a nuestros migrantes temporales internacionales a una vejez de pobreza y frustración, como se puede apreciar en la mayoría de los ex migrantes ya no aceptados o jubilados.

Los tristes resultados obtenidos en 2013 , cuando el único notario de la ciudad de Tlaxcala se negó a constituir una asociación civil de representación que quiso hacer un grupo importante de migrantes; y en 2014 cuando la SAGARPA no aceptó ningún proyecto productivo de migrantes independientes de organizaciones político sociales, conducen a pensar que los habitantes del campo continúan siendo solamente un botín político, un reservorio de votos esencialmente para el partido en el poder y sus aliados. Estos requerimientos del Estado obligan a buscar el apoyo de dichas organizaciones para poder contribuir a mejorar la precaria situación económica y social de los migrantes tlaxcaltecas del PTAT.

Así, en 2015 tenemos ya aprobado un proyecto, a través de la Unión Campesina Democrática. Esto podría abrir opciones a futuro, pero no para los migrantes como tales, sino como afiliados a una organización política que finalmente, al igual que todas las organizaciones de este tipo en el país, son las únicas autorizadas a conseguir los recursos del Estado para la reactivación productiva del campo.

\section{BIBLIOGRAFÍA}

BERUMEN, S. y López, J. A. (2012) Pobreza y migración, Colección Migración, SPMAR, UPM, INM, Tilde, SEGOB. 
COMISIÓN DE LAS COMUNIDADES EUROPEAS (2008) Una Política Común de Emigración para Europa: Principios, medidas e instrumentos, Bruselas 17.6.2008, en internet Eur-Lex52008DC0359

DÉCOSSE, A. (2012) Reflexionando el utilitarismo migratorio: los jornaleros marroquíes bajo contrato OMI en Francia (IRIS/ EHESS), Conferencia presentada en CEMCA, México D. F., 21 de abril del 2012.

DRUCKER, P. (1994) La Sociedad Post Capitalista, Barcelona: Grupo Editorial Norma.

GONZÁLEZ, M. E. (2008) La migración temporal como mecanismo de transferencia del bono demográfico. El Programa de Trabajadores Agrícolas Temporales México - Canadá en Tlaxcala, Tesis de Maestría en Desarrollo Regional, El Colegio de Tlaxcala.

HERMAN, P. (2003) El gusto amargo de frutas y legumbres, en Le Monde Diplomatique http://monde-diplomatique. es/2003/04/herman.html, consultado el 30 de enero de 2014.

INMUJERES (2014) Directorio de programas institucionales dirigidos a la población migrante, en http://cedoc.inmujeres.gob.mx/documentos_download/ DirectorioMigrantes2014.pdf, p. 42, consultado abril de 2015.

MUÑOZ, L. M. (2011) Programa de Trabajadores Agrícolas Temporales México - Canadá: Costos y Beneficios, The George Washington University, en http://www.gwu.edu/ ibi/ minerva/Spring2011/Luis_Munoz_Spanish_version.pdf, consultado el 14 de febrero de 2014.

POY, L. (2012) Alto grado de vulnerabilidad enfrentan los trabajadores mexicanos en Canadá: expertas, en: La Jornada 12 de abril de 2012, Año 28, Número 9941, p. 17.

PREIBISCH, K. y Binford, L. (2007) Interrogating Racialized Global Labour Supply: An Explanation of the Racial/National Replacement of Foreign Agricultural Workers, Canadian Review of Sociology and Anthropology.

REIGADA, A. (2012) Agricultura industrial en Andalucía y feminización del trabajo en las cadenas agrícolas globales, Revista Regiones, suplemento de antropología, número 47, enero-marzo de 2012, pp. 22-26.

SEPÚLVEDA I.; ROMO, B., BARRANCO, S. y GONZÁLEZ, M. E. (2012) Trabajo mexicano en la agricultura canadiense, Castellanos Editores.

SEPÚLVEDA, I. (2012) Dos rutas a Canadá, Conferencia en el Seminario Permanente de Migración México - Canadá, UNAM, 3 de octubre de 2012

SER, s/f: Seguro de Desempleo y Prestación de Paternidad, Consulado General de México, Montreal, Canadá, en http:// consulmex.sre.gob.mx/montreal/images/Consulado/ PTAT/segdesempleopaternidad-ptat.pdf, consultado el 2 de marzo de 2015.

STPS (2012). Programa de Trabajadores Agrícolas Temporales México-Canadá. Presentación del Lic. Luis M. Muñoz C. en el Espacio de Reflexión Sobre las Políticas Laborales Canadienses. Muñoz de Domingo Arenas, Tlax. México.
STPS (2014) Portal de empleo. http://www.empleo.gob.mx/ en_mx/empleo/ acuerdo_de_cooperacion_laboral_mexicocanada_, consultado el 3 de marzo de 2014.

THOMAS, M. (1997) The Search for a "Reliable Workforce"; The Canadian State and the Seasonal Agricultural Workers Program, Thesis, The Department of Sociology and Anthropology of the University of Guelph, Canada.

\section{NOTAS}

1 El mismo vuelo solicitado en septiembre de 2015 por internet a Despegar.com, cuesta 496.78 dólares canadienses

2 Información proporcionada por Margarita Cisneros Tzoni, Delegada del Servicio Nacional de Empleo en Tlaxcala, durante su presentación en el evento "Espacio de Reflexión sobre las Políticas Laborales Canadienses" organizado por Chapingo y realizado en Muñoz de Domingo Arenas, Tlax. 1 y 2 de marzo de 2012.

3 Básicamente consta de dos mujeres entusiastas, que dedican muchos fines de semana a conversar y recoger información con grupos de migrantes y sus familias, que otras mujeres, migrantes o esposas, citan en sus casas. A este se unió un joven ingeniero agrónomo miembro de un despacho privado de asesoría técnica, que busca su nicho laboral con los eventuales proyectos que logren los migrantes.

4 Se intentó crear una asociación civil, pero el Notario $N^{\circ} 1$ de Tlaxcala, Tlax., se negó a darle formalidad a esta figura que ya tenía autorizada su razón social y para la cual los jornaleros ya habían juntado el dinero necesario. Sus razones quedan a la imaginación. 